\title{
A computer support tool for the early stages of architectural design
}

\author{
Dzmitry Aliakseyeu *, Jean-Bernard Martens, Matthias Rauterberg
}

User Centered Engineering Group, Faculty of Industrial Design, Eindhoven University of Technology, Den Dolech 2, P.O. Box 513, 5600MB Eindhoven, The Netherlands

Received 16 June 2004; received in revised form 3 November 2005; accepted 7 November 2005

Available online 4 January 2006

\begin{abstract}
Even in the current computer age, there are still many important application areas, such as early architectural design, where traditional tools like sketching on paper continue to be preferred by many professionals over computer-based tools. There is a growing awareness that there are often very good grounds for this preference. Hence, instead of trying to replace such traditional ways of working, it is now often considered more opportune to try and preserve the strengths of these traditional ways of working, while at the same time improving them by providing access to new media. This is one of the main objectives of the augmented reality approach that we adopt here. In this paper, we specifically discuss the realization of a tool for early architectural design on an existing augmented reality system, called the Visual Interaction Platform. We describe the development process, the resulting tool and its performance for elementary tasks such as positioning and overdrawing. We also identify directions for future research and applications.
\end{abstract}

(C) 2005 Elsevier B.V. All rights reserved.

Keywords: User-centered engineering; Augmented reality; Tangible interfaces; Architectural design; Electronic paper

\section{Introduction}

Various computer tools have been proposed and are in use today to support the design of new products, including the design of buildings. Much progress has been made in terms

\footnotetext{
* Corresponding author. Address: Den Dolech 2, HG 3.51, P.O. Box 513, 5600MB Eindhoven, The Netherlands. Tel.: +3140247 5245; fax +31402475376.

E-mail addresses: d.aliakseyeu@tue.nl (D. Aliakseyeu), j.b.o.s.martens@tue.nl (J.-B. Martens), g.w.m. rauterberg@tue.nl (M. Rauterberg).
}

0953-5438/\$ - see front matter (C) 2005 Elsevier B.V. All rights reserved. doi:10.1016/j.intcom.2005.11.010 
of product quality and process efficiency since the introduction of computer-aided design and computer-aided manufacturing systems. Most of these programs accomplish this improved support by providing extensive functionality in the form of pre-defined functions and objects, such as generic construction elements, that can be tailored to a specific purpose by specifying lists of parameters. Because of the geometric precision and the large number of detailed selections that they require, such systems are not very well suited for early design. Indeed, the required level-of-detail, although necessary for the operation of these programs, is often largely irrelevant and tends to distract from the design activity itself. In order to get proficient with such systems, i.e. to reduce the cognitive load imposed by their operation, users also require extensive training and frequent practice. Advanced computer tools thereby typically enter only at a later stage in the design process when many global, but nevertheless crucial, decisions about the design have already been made, and the focus shifts towards detailed specification.

Many architects still prefer to use paper and pen or scale models in the early design stage (Gross and Yi-Luen Do, 1996; Aliakseyeu, 2003). These media offer the required flexibility, speed and natural (intuitive) interaction. This way of working, however, creates an interruption in the design process flow, since the architect has to transfer his/her design into computer-aided design specifications after the early design stage. Therefore, in order to reduce the time spent on the transition from the early design stage to more precise stages, more and more architects start to also use programs like AutoCAD, ArchiCAD, Arc + in all stages. The down side of this practice is that the use of such precise programs in the early design stage tends to limit the creativity and can encourage poor design (Lawson, 1999).

Hence, there seems to exist an obvious need for a tool (or platform) that allows to fluently mix very different interaction styles, such as the traditional 'pen-and-paper' interaction and the WIMP (Windows, Icons, Menus, Pointers) 'computer' interaction. In this way, the flexibility and speed of the traditional working environment might be combined with the power of computer applications. Motivated by the above observations, we adopted as the aim of our research to design, implement and validate a computersupport tool for the early stages of architectural design.

One possible approach towards creating such a new design support tool is through the use of Augmented Reality (AR). AR allows to preserve the characteristics of traditional media, while at the same time augmenting them with access to new functionality. The interaction with such system can be facilitated trough the Tangible/Graspable User Interfaces (TUIs). TUIs allow direct control of electronic objects through physical artifacts, such as bricks. A tangible object is an object composed of both a physical handle (i.e. one or more physical artifacts attached) and a virtual object. From the user's perspective, the physical object acts as a physical handle to virtual objects and offers a rich combination of physical and virtual affordances (Fitzmaurice et al, 1995; Ullmer and Ishii, 2001). Thus, it can potentially make computer-based interactions more intuitive.

Because of the complexity of the architectural design process and the vagueness of the early design stage, it was difficult to specify a priori the requirements for our targeted computer-support tool. Therefore, we have adopted an approach in which frequent (often informal) usability experiments were performed with prototypes. This user-centered engineering approach has helped us to obtain new insights and requirements throughout 
the duration of our project. Based on specifically formulated design guidelines and requirements, we have also designed, implemented, and evaluated the Electronic Paper system. We are hence able to produce evidence for our claim that the final Electronic Paper system does indeed offer a computer interface for (architectural) design that supports much of the freedom, flexibility, abstraction, speed and ease of use of traditional pen and paper. Before describing the design, implementation and evaluation of the Electronic Paper, we first discuss some related work in Section 2.

\section{Related work}

Much research and development has been invested in the fields of computer-aided design and AR. They have resulted in a range of potentially useful functionalities for early design, as well as in alternative and more natural ways of user interaction with such functionalities. We divide the related work into five subsections. First, we provide a short and general introduction to AR and tangible user interfaces. Next, we discuss AR and TUIs that more specifically aim at supporting architects (and designers). Third, we introduce some interesting and innovating sketching tools that have been developed for desktop or tablet computers, but that may prove to be even more useful in an AR environment. Fourth, we discuss the technologies that are most commonly used in AR systems. Last but not least, we pay attention to the few formal evaluation studies that have been performed up to now on related AR systems.

\subsection{TUI/AR systems}

Within AR, the focus is on improving the human-computer interactions required for operating the functionalities offered by computer programs, rather than on improving the functionalities themselves. The DigitalDesk (Wellner, 1991) is one of the earliest AR systems. It consists of a desk with a computer-controlled camera and a projector above it. The camera observes where the user is pointing, and reads portions of documents that are placed on the desk. The projector provides feedback by projecting electronic objects onto the desk surface. The DigitalDesk adds electronic features to physical paper, while physical objects can be used to operate electronic documents. The system allows a natural user interaction with paper and electronic objects by touching them with a bare finger (digit). Instead of 'direct' manipulation with a mouse, this is a tangible manipulation with fingers.

Many examples of related tabletop and wallboard systems can now be found in the AR literature. These systems can be divided into three major categories:

1. systems that use tangible objects to improve the interaction with purely digital information, such as BuildIt (Rauterberg et al., 1997), metaDESK (Ullmer and Ishii, 1997), ActiveDesk (Ullmer and Ishii, 2001), SKETCHPAD+ (Piccolotto, 1998), iRoom (Fox et al., 2000), etc.

2. systems that add digital information to enrich the interaction with physical artifacts like paper, such as LivePaper (Robinson and Robertson, 2001), Collaborage (Moran et al., 1999a, 1999b), RASA (McGee et al, 2002), etc. 
3. combinations of both approaches, such as those found in URP, Luminous table (Ishii et al., 2002), Illuminating clay (Piper et al., 2002), Liveboard (Elrod et al., 1992), Tivoli (Pedersen et al., 1993), Flatland (Mynatt et al., 1999), Designers' outpost (Klemmer et al., 2001), etc.

While AR offers a range of diverse and potentially interesting interaction techniques, the field has been largely dominated up to now by the design of fairly simple proof-ofconcept applications and by solving the technological challenges involved. There is an obvious need for understanding the potential impact of such systems within specific and more complex practices, such as architectural design. To a large extent this impact will be determined by how well such new systems can be integrated with or can complement existing ways of working.

\subsection{Design related $A R / T U I$}

Some of the above mentioned tools were specifically conceived to support architects and designers within the early stages of the design process. These applications are therefore specific examples of how computerized tools can enrich early design.

Limunious table (Ishii et al., 2002) for example combines and integrates twodimensional (2D) drawings, physical models and digital models to support the urban design process. It uses two cameras to detect the positions of three-dimensional (3D) physical models that represent building components. Two projectors are used to create dynamic digital simulations onto the table surface. More specifically, the software of the system allows to dynamically simulate sun-shading, traffic movements, wind patterns and so on.

Designers' outpost (Klemmer et al., 2001) combines Post-It paper notes with a large physical workspace (i.e. a SMART board) and electronic media in order to support web design. By means of this tool web designers can collaboratively work on the information architecture of a web site.

SKETCHPAD + (Piccolotto, 1998) applications focus on creating 3D scenes in a natural and intuitive way on systems that only offer $2 \mathrm{D}$ interactions. It is based on interpreting 2D pointer (pen) movements as movements on surfaces in 3D space. SKETCHPAD + is a prototype system that uses a large design table that is both a penbased digitizer and a computer display, and that resembles the traditional drawing board. The users of SKETCHPAD + can sketch into photo-realistic simulations of architectural spaces, while the system also supports collaborative work by allowing several designers to work simultaneously on a single model.

\subsection{Sketching tools}

Another class of applications that supports architects within the early stage of the design, without resorting to AR technology, are sketching tools such as Cocktail Napkin (Gross and Yi-Luen Do, 1996) and SILK (Landay and Myers, 2001).

The electronic cocktail napkin (Gross and Yi-Luen Do, 1996) prototype is a computer application that allows the kind of informal freehand drawings that designers make during 
conceptual design. It additionally supports the editing and managing of diagrams and drawings, and can be trained to recognize and interpret simple patterns.

Architectural studio is another desktop application that simulates a conventional desk by means of virtual tools that resemble real tools such as pencils, markers, erasers, and tracing paper (Novitski, 2002). The screen, like a conventional desk, can be filled with imagery from many sources: scanned photographs and hand-drawn sketches, drawings imported from other projects, stacks of alternative concept sketches, renderings, etc. With the tracing-paper tool, architects can overlay a translucent 'trace' and can develop new variations of a design while preserving old ones. Sketches made within this environment look very much like sketches that have been hand-drawn, scanned, and imported. This tool also allows exporting the created designs into advanced computeraided design programs.

SILK (Sketching Interfaces Like Krazy) (Landay and Myers, 2001) is an informal sketching tool for graphical user interface design that combines advantages of paperbased sketching with electronic tools. Using SILK, a user can quickly sketch an interface using a digital tablet and pen. The system attempts to recognize the drawn interface elements and adds functionality to the recognized interface elements. This permits to explore the behavior of the drawn interface elements while they are still in the 'sketch' state. When the designer is satisfied with a result, SILK can convert drawn interface elements into real widgets and graphical elements.

There are also several commercial software products, such as Painter (by Corel, www.corel.com) and SketchUp (from Last Software, http://www.sketchup.com/) that support two-dimensional and three-dimensional drawing in a more natural and flexible way.

Our Electronic Paper system will be similar in functionality to the above systems, but will concentrate on further reducing the distinction between real and virtual tools by including more natural AR interaction techniques.

\subsection{Technologies}

Existing AR systems differ in the technologies that are used for registering and tracking user actions. Vision-based tracking is most commonly used for tracking physical objects (BuildIT (Rauterberg et al., 1997), LivePaper (Robinson and Robertson, 2001), etc. Other technologies that are available for this purpose are ultrasound, magnetic, RFID. Interactions with flat computer screens can also be facilitated by using touchsensitive surfaces such as DiamondTouch (Dietz and Leigh, 2001) or Smartskin (Rekimoto, 2002).

For sketching, digital boards, such as Wacom (www.wacom.com) tablets or CalComp (www.calcomp.com) drawing boards, are most commonly used. The video-based pen ANOTO (http://www.anoto.com/) can be used as an alternative sketching tool.

The technology used for visualization is mostly dictated by the size of the display. Touch-sensitive LCD displays are relatively small-sized, and have the advantage that they can easily be made portable. Larger display sizes are most frequently implemented using front or back projection. 


\subsection{Evaluations}

Only a few formal evaluations of related AR systems have been reported in the literature up to now. One noticeable example is the evaluation of the RASA system (McGee et al., 2002). This system augments the paper tools that are used in commands posts (large map with Post-Its that represent different military units) with digital information. The evaluation confirms that the AR system is as easy to use as the original paper-based system. Most users preferred RASA because it provided an easy access to computing functionality, and because it did not replace but rather complemented physical paper.

Another comparison study was done by Elliot and Hearst (2000, 2002). They compared the digital desk, the desktop PC and the tablet PC for sketching and image sorting tasks. Their experiment showed that, for the sketching task, the digital desk and the tablet PC were equally preferred over the desktop PC, but that the quality of sketches was better for the small tablet. The difference in quality was mainly caused by the parallax of the digital pen for the digital desk. For the image-sorting task, the desktop PC was preferred. Using an interaction technique adopted for large displays instead of the currently used drag-anddrop technique to perform this task on the digital desk might change this situation, though.

More general studies involving graspable (tangible) interfaces were performed by Fitzmaurice and Buxton (1997). The results of their experiments indicate that spacemultiplexed, specialized, graspable devices (each function to be controlled has a dedicated physical transducer) outperform mouse-based, time-multiplexed, devices (one physical device is used to control different functions). Their results also suggest that it may be faster to acquire another attached device that is out of reach rather than to attach a virtual control with a device already in the hand.

With our Electronic Paper tool, we intend to explore the potential of AR in more depth, by (1) creating a more extensive AR application targeted at a fairly specific task and user group, and by (2) evaluating and improving this application based on both informal evaluations and more formal experimental validations.

\section{Electronic paper}

This section describes the requirements gathering as well as the design, implementation and evaluation of the Electronic Paper prototype.

\subsection{Requirements gathering}

We started by identifying steps in the design process that can potentially be strengthened and improved by means of computer tools, and by formulating requirements for the targeted computer-aided design tool. First, we studied the domain of architectural design, with a focus on conceptual design, in order to get a better understanding of our targeted user and the context of use. We used a literature study, combined with observations and a questionnaire with 20 practicing architects, to accomplish this. As a result, the high-level task of designing was decomposed into more basic tasks, such as 
sketching, presentation, finding information, etc. Next, the relevant developments in the fields of computer-aided design and AR were reviewed. This review helped to identify important interface requirements (or opportunities for improvements) and to specify in more detail the potential added value of computerization. Based on this analysis, we narrowed down our interest to developing an interface that can be common to traditional design activities and emerging, computer-supported, activities.

Our main resulting design guideline can be formulated as follows: the architect must be able to sketch, write, perform 3D modeling and search for images, or other relevant information, in an easy and intuitive way. Intuitiveness can be provided to architects by a tool that is natural and familiar to them.

\subsection{Design and implementation}

In this section we discuss the detailed considerations that were involved in the evolution towards the final Electronic Paper prototype. We discuss both software and hardware aspects. We especially highlight the design alternatives that were considered and justify the particular solutions that were chosen.

\subsubsection{Design considerations}

We have organized the discussion along the major design aspects involved, i.e. the data types to be incorporated, their representation and their manipulation.

Data types. Previously, we noted that the ability to sketch is crucial for designers. Besides sketching, architects also use additional materials that can assist or support them in the design process. The literature study, observations and questionnaire showed that images (photos) are considered to be the most important additional supporting material, followed by textural data and 3D models. Therefore, in the Electronic Paper prototype, we concentrated on data types that could contain sketches and images. In addition to sketches and images, we have also included the textual data type. This means that an annotation or description can be added to a sketch or image. In order to limit the complexity of the prototype, it was decided to exclude 3D models for the time being.

Data representations. The data representation was as much as possible inspired by the familiar metaphor of 'pen and paper'. More specifically, the Electronic Paper prototype supports the virtual paper representation. In addition to virtual papers, the prototype also supports a virtual album. The virtual album metaphor serves as an intuitive way of separating different design alternatives, and of grouping related data (images and sketches).

Drawing/sketching. Since sketching is central in the early design stage, the system should provide the user with the ability to sketch in an intuitive and natural way. Intuitiveness in using the program is important in order to enable the architect to focus on the design problem and not on how to use the tool.

The computer-supported workspace was implemented using a Wacom ${ }^{\circledR}$ UltraPad A2sized tablet (active area $635 \times 462 \mathrm{~mm}$, resolution $2540 \mathrm{dpi}$ ), in combination with projection from above. Top projection potentially creates problems with shadows that are cast by hands and other objects, but this solution is less expensive and easier to create with off-the-shelf components. Later experiments and a literature study have demonstrated that 
the problem with covering part of the workspace is not very severe, and that users easily adopt their posture to avoid this problem (Wellner, 1991; Hinckley, 1997; Aliakseyeu, 2003).

Positioning. Another action that the user has to perform frequently is positioning objects within the workspace. The following a priori guidelines for positioning were adopted from the start:

1. two-handed interaction should be preferred over one-handed interaction; If the task context allows to use both hands, people will naturally involve both hands in the execution of handwriting and sketching tasks. Even when the left hand is not directly involved in the manipulation itself, it often plays a role in postural support or maintaining split attention, such as the observed use of the left hand as a place holder when copying text from one page to another. (Guiard, 1987; Hinckley, 1997). Moreover, exclusion of the non-dominant hand from a writing/sketching task reduces the writing/sketching speed (Guiard, 1987; Hinckley, 1997).

2. combined translation and rotation should be preferred over separated actions; An experiment of Fitzmaurice et al. (1999) on artwork orientation showed that while sketching, designers tend to rotate and slightly reposition the paper with their nondominant hand. They also showed that restricted rotation can lead to reduced quality results (Fitzmaurice et al., 1999).

3. wireless positioning devices should be preferred over wired ones; Wires may hinder movements of the input device especially in the situation where several devices are being used simultaneously (Gribnau, 1999).

4. absolute positioning should be preferred over relative positioning. This guideline arises from the general requirement that action and perception space should coincide. The displacement of the display and input surface for drawing and writing tasks produces an eye-to-hand coordination problem. This is claimed to cause discrepancies and unexpected results (Rauterberg et al., 1997; Fitzmaurice et al., 1999).

The fact that we used a digital pen with a drawing tablet for sketching implied that we could not simultaneously use touch-sensitive technologies, such as DiamondTouch (Dietz and Leigh, 2001), for positioning operations. We therefore adopted tangible bits (Graspable/Tangible User Interface) (Fitzmaurice et al., 1995) as our approach to positioning, since tangible bits seemed to fulfil all requirements. The Electronic Paper prototype uses optical tracking by means of a camera to establish the position and orientation of brick-shaped objects in the workspace. In order to enable high recognition speed and robustness, the use of infrared lighting and infrared-reflecting markers was adopted. Up to 32 infrared tagged tangible objects can be tracked simultaneously. These objects can be operated by multiple hands and/or by multiple users.

The results of a preliminary prototype evaluation, in which only simple brick-shaped tangible objects (that are called brick elements) were available for positioning, revealed that they are not always well suited for an easy and seamless positioning, especially while sketching is performed. Based on the results of this evaluation we added a number of requirements to our initial list, i.e. 
1. the brick elements need to be designed in physical appearance (shape and affordance) so that incorrect use, such as unintentional covering, is minimized;

2. the brick elements should also receive visual feedback that reflects when and where they are observed by the system;

3. some of the tasks related to positioning, especially the ones that require high precision, can probably be performed better with the pen; therefore, it was decided that a range of positioning techniques that trade speed versus accuracy should be supported;

4. it was judged acceptable that users might need some initial training in order to learn how to best handle brick elements before starting to work with the Electronic Paper prototype.

Until now, little has been done to redesign the physical appearance of the original brickshaped tangible objects, except for adding physical papers with infrared markers, called enhanced physical papers, as new positioning tools. More specifically, enhanced paper can exist in different sizes (such as A4 and A5) and contain two rectangular markers. One of the markers is long and elongated to improve the estimation of the paper orientation. Two markers that move coherently are interpreted by the system as evidence for an enhanced paper. Because of the importance of physical pen and paper in current architectural design, augmenting paper seems a promising approach towards improving paper handling (Mackay and Fayard, 1998; Aliakseyeu and Martens, 2001; Holman et al, 2005). Different virtual objects can be mapped on top of a paper and a (real or digital) pen with ink cartridge can be used to draw on it. The enhanced papers do not fully replace the original rectangular brick elements, since many selecting and positioning operations that do not require high precision are easier to perform with a small brick. Because of the smaller size of these bricks, it is easier to manipulate several of them at the same time.

The Electronic Paper has an alternative way of positioning objects on the workspace. The underlying idea is that the user can use tangible objects for positioning objects during drawing when high precision is not essential. In other cases, such as when aligning virtual objects, high precision may be required. If the dominant hand is available, the digital pen with its much higher spatial accuracy, can be used for positioning or fine-tuning. Two different positioning techniques with the digital pen have therefore been included-dragand-drop and pick-and-drop (Rekimoto, 1997). With drag-and-drop, users select a virtual object and translate it with a pen without releasing the pen from the surface. A drag-anddrop operation on one of the corners of a virtual object can also be used to resize and rotate it. In the case of pick-and-drop, users select a virtual object by first touching it with the digital pen and then lifting the pen. The selected object disappears and only reappears when the pen is moved close to the surface. As the user touches the surface again the object is dropped at that location. Pick-and-drop has some advantages over drag-and-drop since it allows to move virtual objects over physical objects that are placed on the workspace. On the other hand, pick-and-drop is only used for translation, and not for resizing and rotating.

Attributes of virtual objects. Most virtual objects in the prototype have attributes that can be adjusted by the user. In order to realize such adjustments, a semitransparent movable function menu can be used. This function menu was inspired by the ToolglassTM transparent interface (Bier et al., 1993). It cannot only be moved freely across the workspace, but also it can provide context-dependent (data-type dependent) functionality, 
and because it is semitransparent, the function menu options and the objects on which the functions are applied can be observed simultaneously.

Additional interaction elements. In order to improve frequent manipulations such as overdrawing and creating collages, virtual pins, clips and an eraser have been added to the original set of virtual objects.

\subsubsection{Implementation hardware}

The Electronic Paper prototype was implemented on an existing tabletop AR platform, i.e. the Visual Interaction Platform (Aliakseyeu et al., 2001) (see Fig. 1). The Visual Interaction Platform is an extension of the BUILD-IT (Rauterberg et al., 1997) system and supports different natural interaction techniques such as writing, sketching, and the

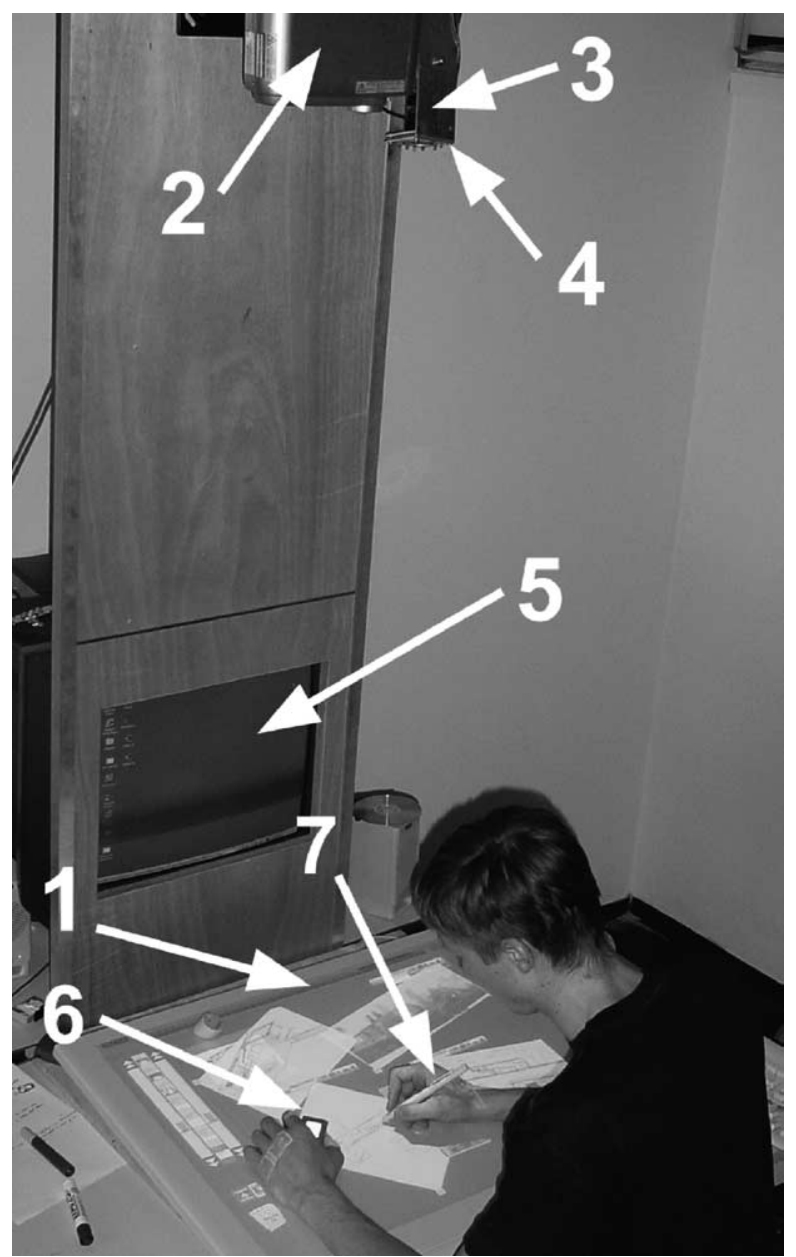

Fig. 1. Visual Interaction Platform. (1) Wacom Tablet (action-perception space); (2) LCD projector; (3) camera; (4) infrared light source; (5) monitor (communication space); (6) brick element; (7) digital pen. 
tracking of objects in 2D space. The Visual Interaction Platform uses an LCD projector (Toshiba TLP-B2ultraE, with a spatial resolution of $1280 \times 1024$ pixels) to create a large computer workspace on a horizontal surface. The horizontal surface on which the projected image is displayed is an UltraPad $\mathrm{A} 2{ }^{\circledR}$ digitizer tablet. This tablet can accurately record digital pen movements, and hence allows the user to perform precise actions in the horizontal workspace, which are necessary for handwriting, drawing and sketching, as well as for making menu selections. The horizontal workspace will be referred to as the action-perception space, since the visual feedback of the pen action is perceived at the position of the pen tip. The system also contains an infrared light source and a black and white camera (Baxall CD6231, resolution $720 \times 576$ pixels, $50 \mathrm{~Hz}$ interlaced). This allows the system to track physical objects that are coated with infrared-reflecting material. These tangible objects can be used for selecting and positioning virtual objects in the working space. The system can distinguish different objects by their size (width and height) and/or by a pattern of holes in the infrared-reflecting material. The Electronic Paper prototype can moreover distinguish identical tangible objects by relying on the history (i.e. the position of all tangible objects are kept in the history, and a nearest-neighbor rule is used to associate the objects in the current video frame with objects in earlier video frames).

Apart from the horizontal action-perception space, the Visual Interaction Platform has a second, vertically oriented, workspace (communication space). This optional second workspace is usually used to supply the user with more extensive visual feedback for increased spatial awareness, or to communicate with remote participants.

The hardware within the system is hence similar to that in many existing tabletop AR systems, i.e. DigitalDesk (Wellner, 1991), BuildIT (Rauterberg et al., 1997), metaDESK (Ullmer and Ishii, 1997) and so on. The main distinction is that the Visual Interaction Platform combines an electronic sketching board with optical tracking of tangible bits. This allows a combined use of very precise pen input, to support operations such as sketching, with less precise but more flexible input through tangible objects.

\subsubsection{Implementation software}

This section describes the layout and interaction of the Electronic Paper prototype in more detail.

We use Microsoft Visual $\mathrm{C}++$ as the software development tool. For visualization and image analysis we use OpenGL (http://www.opengl.org), and Intel OpenCV libraries (http://www.intel.com).

The layout of the action-perception space in the prototype (see Fig. 2) consists of virtual clips and pins $(1,2)$, an image database browser $(3,4,5)$, a function menu (6), virtual papers (7), and the visual feedback of brick element (8).

The image database browser is located in the left margin of the action-perception space and consists of several images that the user has previously acquired, either through this interaction tool or through other means. The browser contains an image database selector (3), image thumbnails from the selected database with two buttons and a scroll bar (4) for browsing, and a preview window (5) that shows a high-resolution version of the currently selected image. Each database corresponds to a predefined directory on the computer system, and images that are transferred into this directory will automatically appear in the image database browser. 


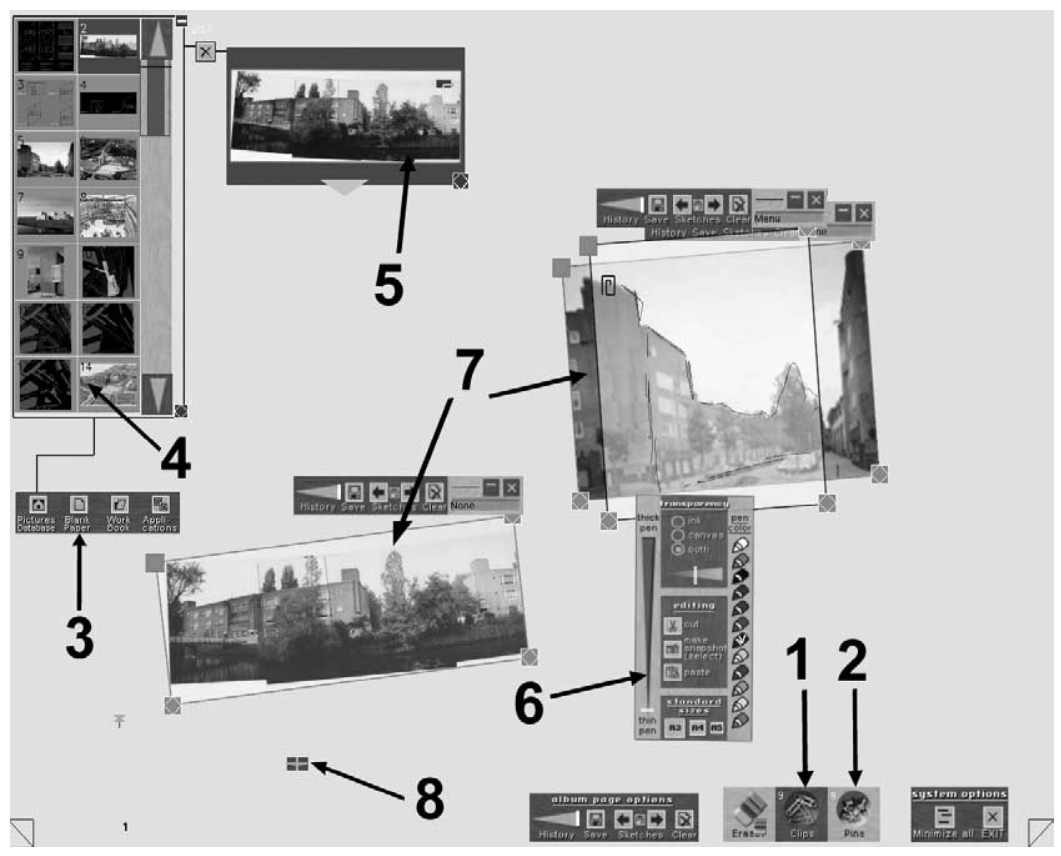

Fig. 2. Layout of the action-perception space of the Electronic Paper prototype. (1) Virtual clips; (2) virtual pins; (3-5) image database browser; (6) function menu; (7) virtual papers; (8) visual feedback of brick element.

A brick element positioned on the scrolling arrows or on the thumbnails can be used to scroll through and select from the database, respectively. The digital pen can also be used to perform the same actions. In addition, the pen can be used for changing the size of the browse menu and for browsing with the help of the scroll bar.

A copy of any image in the browser can be selected and dragged (with a brick element) or picked and dropped (with the digital pen) from the browser into the working area, hence creating a virtual paper. A virtual paper can also be created through the windows clipboard. The image copied into the clipboard from any windows application can be pasted into Electronic Paper prototype as a virtual paper. The reverse action is also possible. Any virtual paper (or part of it) together with a sketch can be placed into the windows clipboard and later retrieved in any windows application. The number of virtual papers that can be placed on a workspace simultaneously is limited only by the computer power (currently the system supports up to 20 virtual papers, in order to keep the frame rate at $40-70 \mathrm{fps})$.

Visual feedback is always provided on the surface of a brick element. This visual feedback depends on the position of the brick element and suggests the actions that can be performed with it in that position. For example, if a brick element is positioned on a thumbnail, an animated arrow shows that the user can now drag the image outside the browse menu.

By using either a brick element or a pen, the user can orient and position a virtual paper in the working area. The digital pen can also be used to sketch and write on the virtual 
paper. All sketches created on any paper can be saved and restored. Every sketch also has a time function, so the user can explore how the sketch evolved through time.

A virtual paper has several properties like transparency level, size, pen color, pen thickness, etc. To adjust the above-mentioned properties, the user can use the function menu. Users can select and drag the menu using either a brick element or the pen. When any of the four corners of the function menu moves within the boundaries of the paper, the menu operates on that paper, thereby enabling the user to change its properties. The properties can be changed by making menu selections with the pen.

The Electronic Paper prototype supports several additional tools like clips (for merging different virtual papers), pins (for attaching a virtual paper to the workspace), and an eraser (to erase parts of a sketch). The virtual pin allows the user to fix a virtual paper on the workspace. Virtual papers with one pin can no longer be translated, but can still be rotated. Papers with two or more pins are completely fixed. The virtual clips allow to attach virtual papers to each other and to the enhanced physical paper. This function can be used to group different papers together, since papers that are attached to each other move coherently.

The virtual album introduces several additional functionalities within the prototype. First, the visible workspace can be used as one big album page (or canvas), which means that it can be used to sketch and write on. An album page has almost the same properties as a virtual paper, i.e. transparency, pen color and thickness, etc. Second, when the user flips an album page forward, a new empty album page appears. The previous page can still be observed if the user decreases the opacity of the current album page. If the album page is flipped back, then the previous page content is restored (sketches, virtual papers, etc.). In order to transfer images from one album page to another, the user can use pins, since pinned virtual paper will not be removed if the user flips to the next or previous page.

Auditory feedback is provided to the user in situations where visual feedback is not clear or not possible. For example, when the user saves a sketch, a sound is played to confirm that the action has been performed successfully.

The Electronic Paper supports collaborative work in a limited way. Several users can observe the interactions and use different tangible objects. Although different users may have separate digital pens, only one can be operated at a time, due to hardware limitations within the Wacom tablet.

The Electronic Paper tool can also be used on a standard desktop with a mouse or digital pen, and on a Tablet PC with a digital pen. Obviously the tangible object-based interactions are not available then. Since almost all functions within Electronic Paper can also be performed with a pen this is not a serious restriction on the available functionality.

The sequence of snapshots presented on Fig. 3 illustrates step-by-step the interaction with a system.

\subsection{Scenario}

In order to provide a concrete example of how the Electronic Paper application could be integrated in the architects working environment we present the following scenario (Fig. 4). Scenarios (Carroll, 1995) help to identify important aspects, such as mobility, 


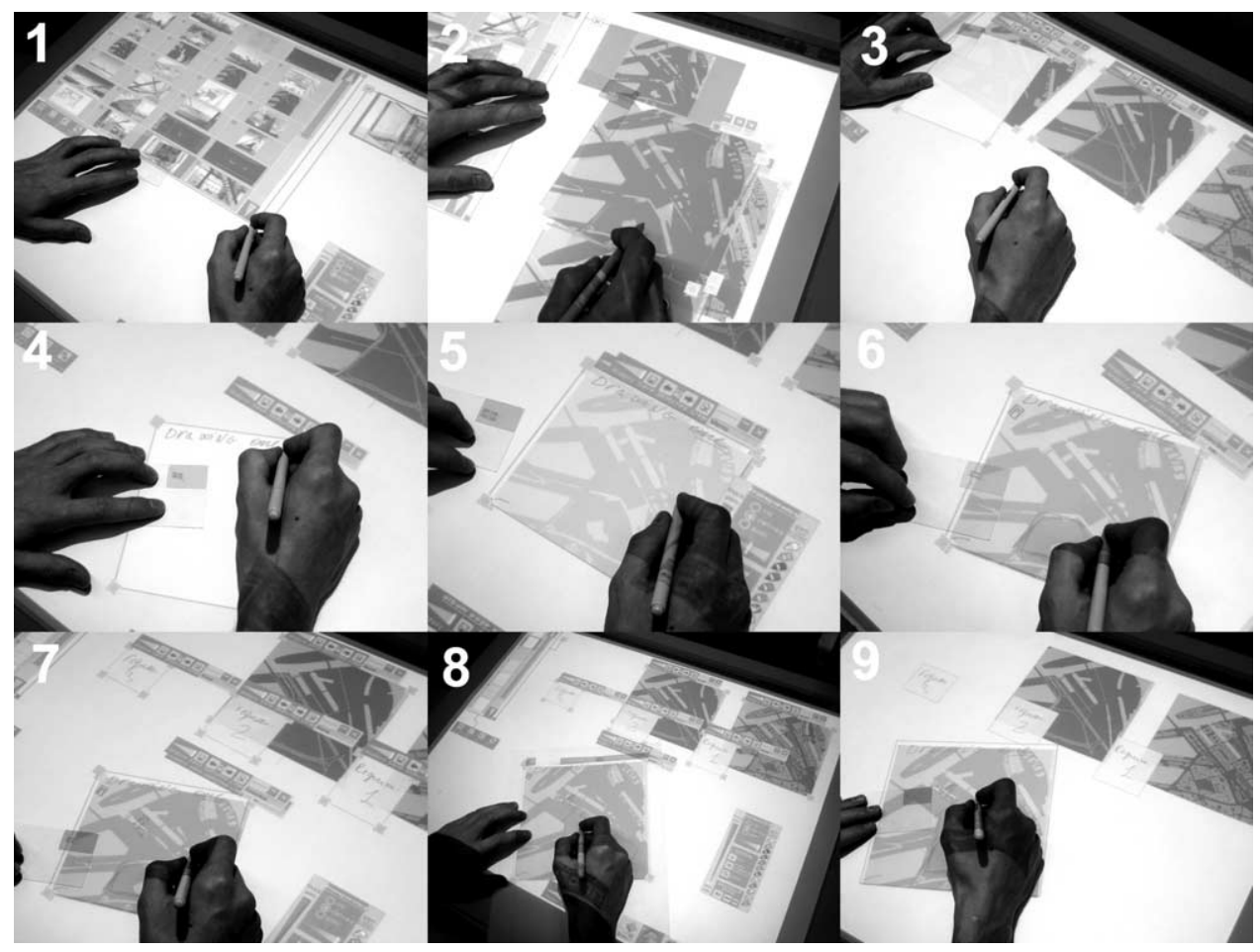

Fig. 3. (1) The image database browser is enlarged to help in the finding of relevant materials. (2) Scheme of the site and some other relevant materials are dragged out of the image database. (3) The image database browser reduced to it original size, then a blank virtual paper is created. (4) Virtual paper is positioned with a brick element and pen is used to annotate it. (5) Blank paper is resized to the size of the scheme of the site and positioned on top of it. (6) After adjusting the transparency two virtual papers are attached with a clip. An architect continues to draw using the underling image as a background. (7) Several Post-IT sized virtual papers with written notes are added to remind of some additional requirements. (8) Using a clip, two virtual papers are attached to the enhanced physical paper that can now be used to position these virtual papers. (9) All interface elements are minimized to make the workspace less cluttered. The architect can continue to work in this mode or can create a snap shot of the workspace without interface elements.

personalization, importing/exporting material, etc. that play an important part in integrating the Electronic Paper application into an actual work environment.

Not all the functionality that is described in this scenario is currently available in the Electronic Paper, although the core components do exist today. More precisely, the Electronic Paper does not support automatic recognition and data transfer between the tablet PC version and the Visual interaction platform version. Currently, this can only be done manually. Also an extended search engine is not yet implemented, and exporting Electronic Paper sketches into other applications formats is not yet possible. Section 5 will describe recent work that we think will help to address some of these issues.

Nicole is a young architect who works in a small company, and who just received an assignment to design a fully detached house for a large family. She first holds a meeting with the client to discuss the assignment and draws up a brief as a summary of the meeting. 


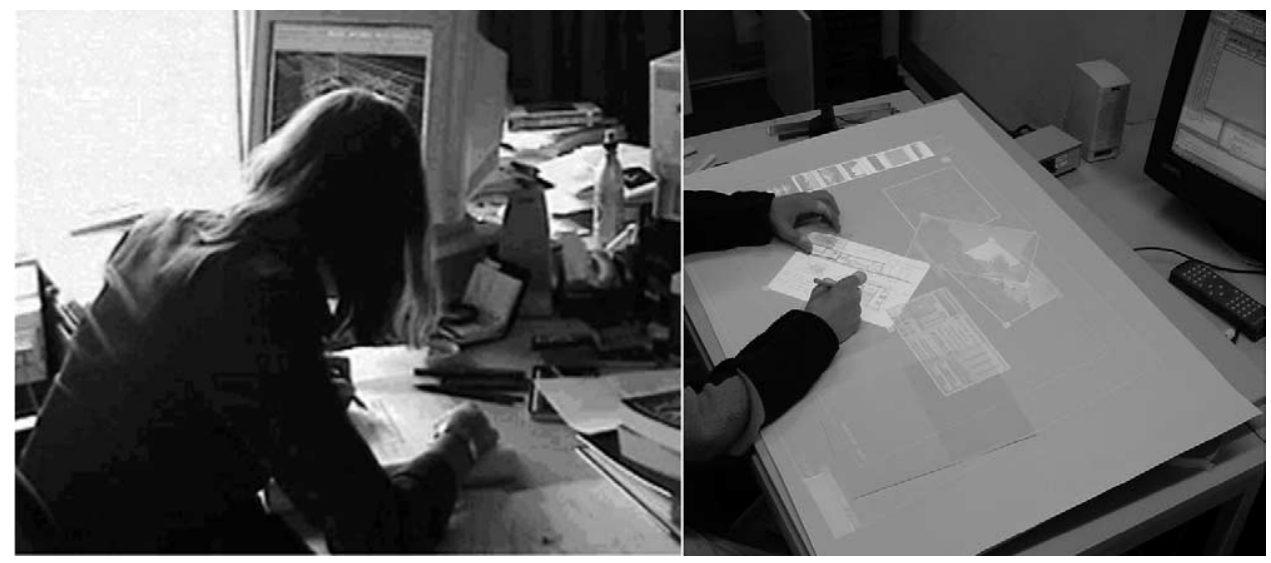

Fig. 4. Traditional environment of the architect (left) and the Electronic Paper environment (right).

The main constraint for the design is that the site is strictly predefined. Another important constraint is that the client wants a house with no more than two floors. After the meeting with her client, she visits the site. It's a rather small but quite beautiful place near the river. She arrives there with already some ideas in mind. On the site she takes several photos with a digital camera connected to her Tablet PC. She also makes some preliminary sketches on the Tablet PC that runs a pen version of the Electronic Paper application. Upon returning to her office, which she shares with three other architects, she places her Tablet PC on the worktable of the Visual Interaction Platform to load her personal profile on the Electronic Paper (automatic recognition not yet implemented). The Visual Interaction Platform in her room runs the full version of the Electronic Paper application and is shared by all four architects. Sketches and photos from the Tablet PC are automatically downloaded into her personal database (automatic download not yet implemented). As a first step she creates a workbook on the Electronic Paper application for this new project and transfers the photos and sketches that she has created on the site to it. In order to find inspiration and information for the new assignment, relevant materials (photos, sketches, etc.) from earlier similar projects are consulted (Fig. 3(1)). While scanning through this visual material she remembers that she has once come across relevant work of another architect. Using some sketches drawn on the workspace she explains to a colleague what she is after and asks him whether or not he remembers the name of the architect that she is looking for. Since he doesn't, Nicole decides to use keywords to search through the shared database of the architectural bureau (extended search engine not yet implemented). She indeed finds some pictures of the architect that she was thinking of. She assembles the most relevant materials up to now in the workspace and starts making notes and annotations (Fig. 3(2)-(4)). She uses the sketch that she made on site as the background image for new sketches. These new sketches are drawn on transparent virtual papers that are clipped to this image (Fig. 3(6)). While sketching she uses pencils with varying colors. At one point, she decides to summarize the main concepts underlying her first design idea by means of a number of keywords. The word-association tool that is integrated within the system assists her in conceiving new design ideas that she also converts into sketches. 
She adds some small virtual papers with the appearance of Post-IT notes to the workspace, in order to remind her of additional requirements that she needs to check with the client (Fig. 3(7)). At the end of the day, some useful ideas have been created and assembled.

The next morning, the system restores the final workspace from the previous day after Nicole has identified herself. All images, sketches annotations, etc. reappear at the same position where they were left. Today she wants to work out the raw concepts that she has made the day before. First she checks the existing sketches, also investigating how they evolved by playing back the sketch history. She selects two designs as the basis for further development, and uses virtual pins to transfer them to separate empty album pages. She increases the size of the virtual paper with the basic design and creates a number of small blank papers. On these blank papers, she creates sketches for different rooms. These small transparent sketches can be moved around on top of the virtual paper that contains the floor plan of the site. Once she is satisfied with the positioning of the different components, she decides to make perspective drawings that illustrate the design from a different point of view. She creates a new blank paper and switches on the perspective grid for aid in drawing.

Once she is satisfied with her preliminary design alternatives, she wants to consult with her client in order to obtain his feedback on them and to clarify some of the requirements. She removes the interface elements from the album pages and creates a picture file for each of the album pages with a design alternative (Fig. 3(9)). She sends these pictures, together with a textual explanation, to her client by e-mail. They discuss the proposed alternatives over a network connection, and Nicole makes annotations on her album pages while she is conferring. The client particularly likes one of the proposed designs, so she decides to explore it further. She creates a drawing of the floor plan and switches on the grid in order to more accurately judge the spatial constraints (size of the site, room, etc.). After that she exports her sketches into ArchiCAD in order to continue the design in a more strict and precise environment (exporting function not yet implemented).

\section{Evaluation}

The evaluation of the system was carried out in two parts. In the first part we tested the usability of the various interaction components using controlled usability studies. In the second part we accessed the usability and usefulness of the system in a high-level architectural task.

\subsection{Evaluation of low level positioning and sketching tasks}

In order to evaluate detailed aspects of the Electronic Paper prototype, we have carried out a user experiment that addresses the question of how closely it approximates reality in low level positioning and sketching tasks. In this experiment we have compared the prototype with a traditional work environment based on physical paper and pencils. Two types of tasks were evaluated: positioning of images in the workspace, and overdrawing using transparent paper. These particular tasks were chosen based on their importance in the early stages of the design process. 
We wanted to test the following hypotheses for our system: (1) users can perform positioning in the Electronic Paper prototype (by means of the brick element or the digital pen) with the same speed as in case of physical paper; (2) users can perform overdrawing in the Electronic Paper prototype (using virtual papers and the enhanced physical paper) with the same speed as in case of physical pen and paper. These hypotheses relate to our system requirement that we want the prototype to support architects in a way that is very similar to the traditional way of working. We do realize that the positioning of physical objects has obvious advantages over the positioning of virtual objects in an AR system. The user can simultaneously translate and rotate physical objects while moving them on top or above the table. Physical objects are also tactile so they require less visual attention. The Electronic Paper has less flexibility in positioning, since objects can only be dragged with the brick element. We nevertheless hypothesize that these differences may not be significant for the tasks under consideration.

An experimental comparison of sketching was performed by Elliot and Hearst (2000, 2002) on three alternative systems, i.e. the digital desk (which is similar to the Wacom tablet used in the Visual interaction platform system but with an integrated LCD screen), a desktop PC and a tablet PC. The experiment showed that the digital desk and the tablet PC were equally preferred over the desktop PC for sketching, but it also showed that the quality of the sketches was better in case of the small tablet PC. The difference in quality was mainly caused by the larger parallax between the digital pen and the displayed image in case of the digital desk (this parallax is caused by the glass plate that separates the two). The main differences between this experiment and ours are the following: (1) the comparison is against real media in our case, rather than against a (desktop/tablet) PC, (2) subjects are allowed to freely orient and position the papers in all cases, and (3) parallax is not an issue in our system since it combines a tablet with projection.

\subsubsection{Tasks}

The positioning task consisted of sequentially selecting and putting five numbered images into correspondingly numbered, and rotated, rectangles. The images varied in size from $5 \times 7$ to $14 \times 10 \mathrm{~cm}$; the average distance between an image and the corresponding rectangle was $35 \mathrm{~cm}$. In the virtual positioning task, the images were virtual papers. The virtual papers were replaced by real photographs of the same size in case of the physical positioning task (Fig. 5). Every positioning task needed to be performed with real paper and with three different virtual techniques-based on combined translation and rotation with the brick element, and separate translation and rotation with pen drag-and-drop and pen pick-and-drop. When an image was aligned within the corresponding rectangle, a sound signal indicated to the subject that $\mathrm{s} / \mathrm{he}$ could proceed with the next image. The sound feedback that signaled successful completion was triggered by the experiment leader in the real paper condition. Restricted positioning tasks, involving only translation or rotation, were also performed with the virtual techniques, but will not be discussed.

The overdrawing task was performed using three different media-physical paper, virtual paper and enhanced physical paper. In case of overdrawing on physical paper, the sketch had to be copied from one piece of (opaque) paper to a piece of transparent paper. In case of virtual paper, two virtual papers where presented to the user-one with a sketch and 


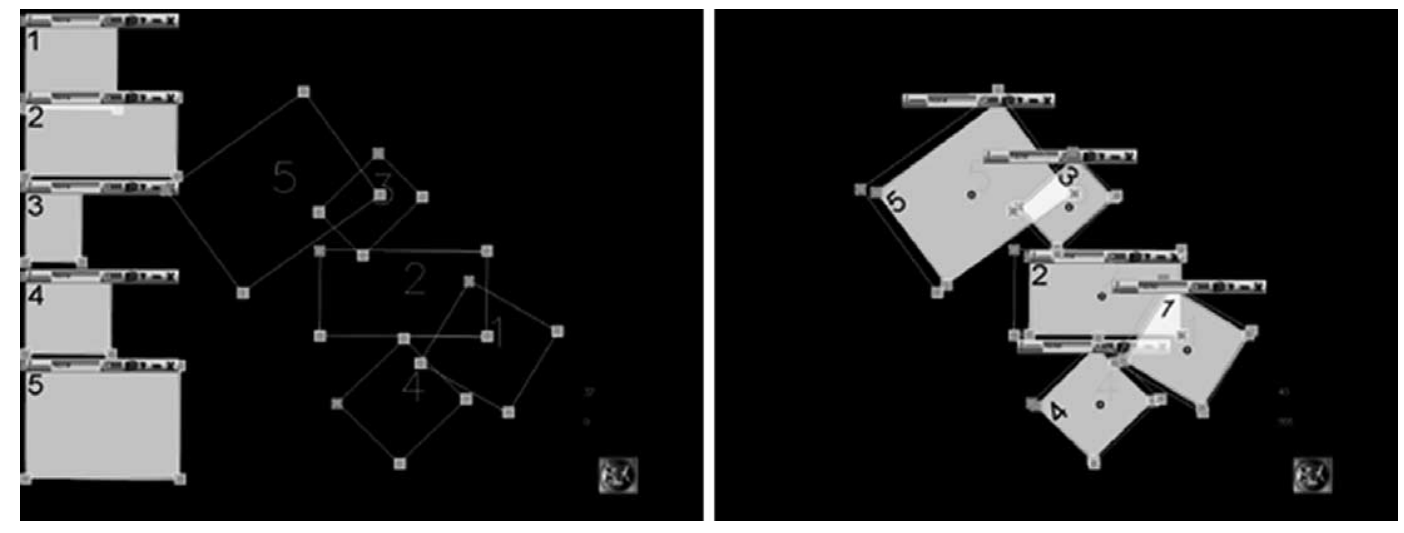


one with blank paper. In case of the enhanced physical paper, an existing sketch on a virtual paper had to be overdrawn on a real piece of paper.

Subjects were requested to fill in a questionnaire immediately after each of the performed tasks. Amongst others, they where asked to rank the different interaction techniques according to subjective preference.

\subsubsection{Experimental design}

All subjects performed the tasks in the same order-rotation, translation, positioning (combined translation and rotation) and overdrawing. A within-subjects Latin square design was used to balance interaction techniques across subjects. Each subject performed all tasks in a single 1-h session with short breaks. The tasks had the same initial conditions with the same number and type of images for all subjects.

\subsubsection{Subjects}

Twenty subjects ( 2 female, 18 male, right-handed with an average age of 22 years) participated in the experiment. Four of them were architectural students, while the rest were industrial design students. All subjects had experience with sketching on physical paper, while none of them had used computer tools for sketching before.

\subsubsection{Results}

Several erroneous trials that were caused by a software malfunction were deleted (2 positioning trials with a brick element and 6 overdrawing trials with the enhanced physical paper). Fig. 6 shows the mean and standard errors of the completion times for all tasks and interaction techniques.

The interaction technique was a significant factor for completion time in case of positioning, i.e. $F_{(3,74)}=7.145$ with $p=.011$ ( 3 , degrees of freedom between conditions, 74 - degrees of freedom within conditions). In the overdrawing task, the medium used was not a significant factor, i.e. $F_{(2,51)}=0.302$ with $p=.741$. This implies that drawing with the enhanced physical paper or on a virtual paper is as efficient as drawing on physical paper.

In the positioning task, all virtual techniques were slower than positioning with physical paper. This is confirmed, for instance, the comparison between the fastest virtual technique, drag-and-drop with the brick element, and the physical technique, i.e. $F_{(1,36)}=$ 8.972 with $p=0.05$. The brick element and pen drag-and-drop had about the same completion time $\left(F_{(1,36)}=0.075, p=.786\right)$, and were $44 \%$ faster than pen pick-and-drop. Since a pen is a familiar tool, especially for designers, subjects felt more confident with this tool than with the new interaction device (i.e. the brick element), which was confirmed in the post-questionnaire. However, the brick element has the advantage that it has one more degree of freedom. With the brick element, subjects are able to simultaneously translate and rotate the virtual paper, while the positioning task needs to be decomposed into two subtasks (translation and rotation) when using the pen. It is well-known from studies performed by Fitzmaurice and Buxton (1997), that space-multiplexed devices (in our case the condition with physical paper) is faster then time-multiplex devices (in our case conditions with a brick element, digital pen drag-and-drop and pick-and-drop).

Pen pick-and-drop, in combination with pen dragging to perform rotation, is clearly the slowest interaction technique for positioning, as is demonstrated by the significant 

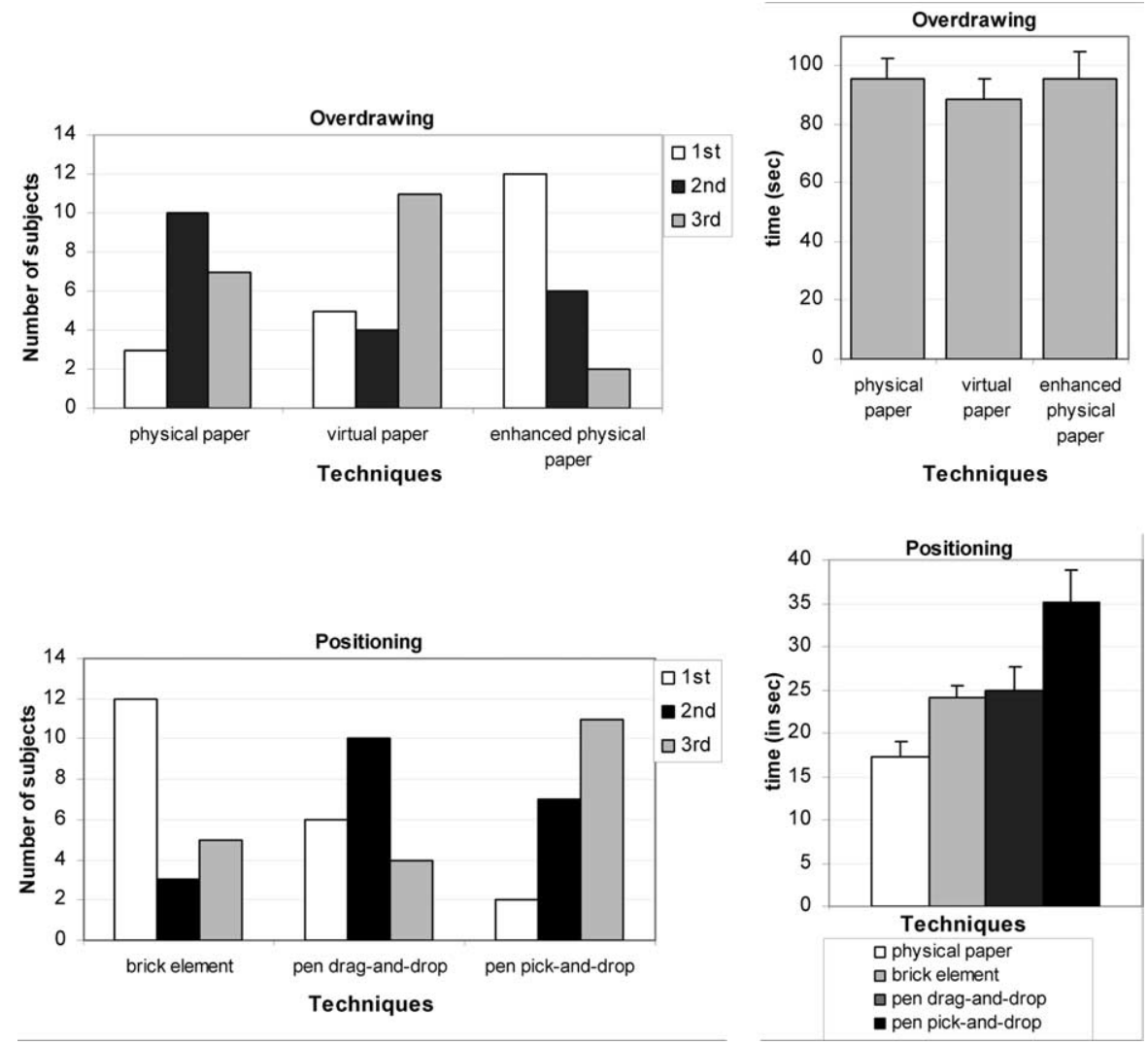

Fig. 6. Left, number of times that an interaction technique was selected as 1 st, 2 nd and 3 rd by subjects in positioning and overdrawing; right, mean completion time for overdrawing and positioning.

difference between pen pick-an-drop and pen drag-and-drop, i.e. $F_{(1,38)}=5.062$ with $p=.03$. There can be several factors contributing to this result. One is that this technique was completely new for all subjects. Completion time for pick-and-drop also demonstrated to be the largest variation between subjects. One subject performed the positioning task in 10 seconds, while another required $65 \mathrm{~s}$. Another factor is that the pen pick-and-drop technique required a long time on target. When using drag-and-drop, the user can continuously observe the position and orientation of the virtual paper, while in case of pick-and-drop, a shadow representing the virtual paper only appears when the pen approaches the tablet. Some delay seems to be involved in visually processing this information.

The order in which an interaction technique was used by the subject did not have a significant effect on the average task completion time (positioning: $F_{(3,74)}=0.484$, $p=.694$; overdrawing: $\left.F_{(2,51)}=0.427, p=.655\right)$.

Fig. 6 shows the number of subjects that rated every technique as first (most preferable), second or third (least preferable). Statistical analysis using a chi-square test confirmed that 
the brick element was indeed preferred in the positioning task $\left(\chi^{2}=9.6, p<.005\right)$ and that the enhanced physical paper was preferred in the overdrawing task $\left(\chi^{2}=9.6 p<.005\right)$.

The fact that the brick element was selected as the most preferable virtual positioning technique is probably due to the fact that this is the only virtual technique that allowed simultaneous translation and rotation. This preference for the brick element agrees with the average completion time measurements.

The enhanced physical paper was the most preferred technique for overdrawing. The overdrawing of (transparent) virtual sketches onto real paper is clearly preferred over overdrawing of real sketches onto transparent paper, and seems to be one of the most appealing features of the Electronic Paper prototype. This was also confirmed by the results of the informal interviews following the experiments.

The subjects found the system easy to use and learn. Subjects had no problems with handling the digital pen, which was confirmed by the post-test interview where all subjects reported that the digital pen was the easiest tool to use and that overdrawing was the easiest task. Most test subjects identified flexibility (in transparency and size) as the main advantage of the virtual paper, in comparison to physical paper, and positioning as its main disadvantage. They found the enhanced physical paper to be a good combination of virtual paper and physical paper, since it can be positioned in the same way as physical paper, while having the flexibility of virtual paper.

Although we did not redesign the physical shape of the brick element but only improved the recognition and visual feedback, the positioning with the brick element was faster than in an earlier preliminary evaluation. In both experiments, the brick element was considered to be the most difficult interaction element to use. Most of the subjects complained about handling the brick element. Especially, the covering/uncovering action required for deselecting/selecting remained unfamiliar. The positioning with the brick element was however, reported as easy, which may be partly due to the well-controlled nature of the task in the experiment.

\subsection{Use of the Electronic Paper tool in a high-level architectural task}

The Electronic Paper prototype was also used as the user interface in an evaluation of a high-level architectural task (Segers, 2002; 2004). The purpose of the study was to measure the effect of a new word-association tool (Idea Space System, described below) on the quality of an architectural design.

\subsubsection{Idea Space System}

The Idea Space System (Segers, 2002; 2004) is a Computer Aided Architectural Design system that is designed to support an architect in the early phase of the design process, by reducing fixation and enhancing the 'flow' of work. The underlying principle of the Idea Space System is that the architect is provided with a structured overview of his/her own ideas - the Idea Space, while designing. The Idea Space System captures all design data (text, sketch, or image), structures the captured information and provides its own interpretation of it. The system might hence be seen as a design partner with an alternative view. The current Idea Space System prototype uses the words entered by the architect to provide real-time (visual) feedback in the form of associations and relations between 
words. The architect can decide whether and how he pays attention to this feedback. It has been demonstrated that such feedback can indeed stimulate the generation of new ideas.

A modified version of the Electronic Paper was used as the user interface to the Idea Space System (see Fig. 7a), because it closely resembles the traditional design environment and it permits to capture information, such as the words being entered (Segers, 2004). This example hence illustrates how the Electronic Paper can serve as an intuitive interface between useful computerized functionality and the user.

\subsubsection{Evaluation}

The subjects were professional architects that had to perform two real assignments, i.e. to design a bus stop and an atrium. In total 19 architects participated in the experiment. In one condition, the architects used only the Electronic Paper, while in another condition, the Electronic Paper prototype was used in combination with the new brainstorming tool. In this particular experiment, only the pen was used (the brick element and enhanced physical paper were not available, because there was no infrared camera in the system) (Segers, 2002; 2004).

A session with an architect took half a day and consisted of general introduction to the experiment, instruction how to use the system (in a form of a tutorial), first trial design session for practicing with a system (about $30 \mathrm{~min}$ ), two actual design session with two assignments (each lasting $1 \mathrm{~h}$ ), and a post-test questionnaire (Segers, 2002; 2004). The observations were done in two ways first using a camera and second using the Electronic Paper itself (all actions that were performed on a system could be observed on a separate computer) (Segers, 2002; 2004).

Based on observations and post-questionnaire results, we can conclude that architects experienced few problems when working with the Electronic Paper. Most of them found the tool useful and easy to use. They also commented that the Electronic Paper prototype is "a quick way to go beyond the standard images and ideas in architectural design; and provides more freedom and creativity". There were two distinct groups of criticisms. Some architects perceived the Electronic Paper prototype as a graphical desktop application and missed additional functionalities that are common for similar applications (such as Photoshop), like selecting part of the image, copy\&paste, etc. Other architects had the impression that the system was an extension of a traditional worktable, and gave completely opposite comments by saying that some of the functions were not strictly necessary, or even distracting, and that the tool could be further simplified.

\section{Discussion and future directions}

This section discusses applications and extensions of the Electronic Paper tool, some of which have already been realized, and some of which still remain to be implemented.

\subsection{VIEWs: Visual Interaction Enriched Windows}

Formal and informal evaluations have showed that by combining digital and physical paper we can make an interaction with only digital paper as fluent as with real paper. 


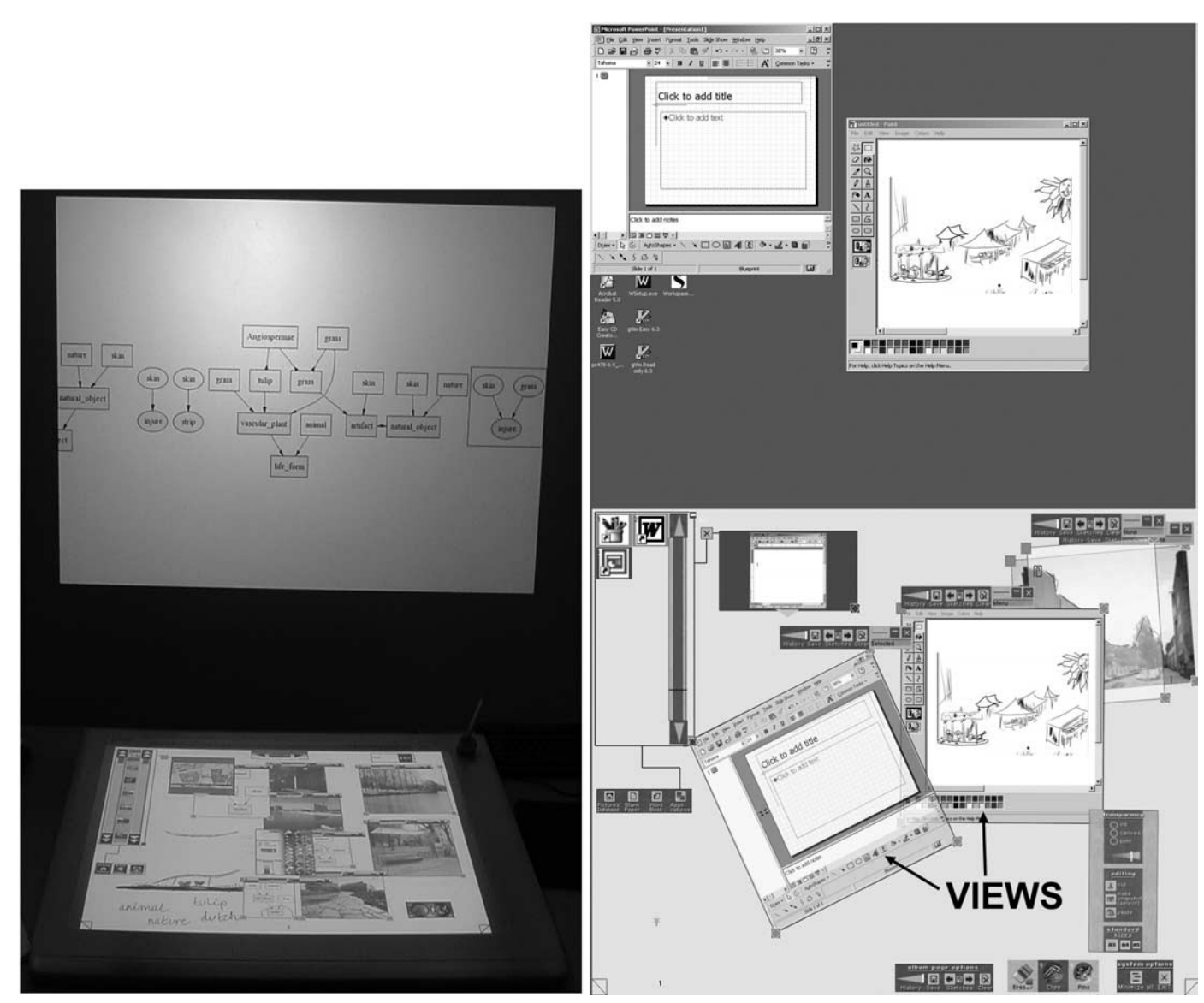

Fig. 7. Left, the Idea Space System; right, the VIEWs, communication (vertical) and action-perception (horizontal) spaces. 
Moreover, we believe that this approach of combining physical paper with digital media transcends application domains and can be used to even augment the traditional computer desktop interaction. Although this combination of physical and digital may not be beneficial to all kinds of computer applications, it definitely might have an added value for some of them (especially graphical applications) and could also improve the integration of different design stages.

With the concept of VIEWs (Visual Interaction Enriched Windows) (Martens et al., 2004), we promote an evolutionary rather than a revolutionary transition from the classical desktop environment to AR environment. In VIEWs, windows applications can still be controlled by standard means, i.e. by using mouse and keyboard. In this way, acquired user skills with existing windows applications can still be exploited. The additional interaction styles that can be offered by an AR platform, including two-handed interaction, pen input and transparency, may, however, be used to improve specific interactions, such as sketching and handwriting, that are more difficult to perform on a classical desktop. The user is free at all times to choose the interaction style that best suits his/her needs when performing specific operations.

The VIEWs concept has been implemented as an extension to the Electronic Paper prototype (see Fig. 7b). The new interaction element (VIEW) was included as an additional component within the prototype. A VIEW has the same properties as a virtual paper, but instead of an image or sketch, the VIEW contains a window application. A VIEW can be created in the same way as a virtual paper by using the image database browser. More specifically, one of the databases in the image browser contains windows applications that are represented by familiar icons. When the user creates a VIEW, the corresponding windows application is started and positioned within the communication space (i.e. the vertical computer screen). Currently, the prototype supports up to three VIEWs simultaneously. A VIEW is dynamically updated by monitoring the video output from the windows application.

The AR capabilities of the Electronic Paper allow for several additional interactions with the windows application. We present a number of these augmented interaction styles, some of which are already available in our current prototype, and some of which still remain to be implemented. The list is obviously non-exhaustive.

First, the Electronic Paper captures all pen events that occur in the VIEW, i.e. the area occupied by the image of the windows application, and maps them to mouse events at the corresponding position in the original windows application. These mouse events will usually influence the visual appearance of the windows application. These visual changes are, however, also reflected in the VIEW that is presented in the Electronic Paper. In this way, the user not only gets the impression that the pen is controlling the windows application, but also receives the visual feedback at the position where the pen is located. This visual feedback at the position where the action is performed is expected to make many operations, especially sketching and writing, easier and more natural. Since all operations in the action-perception space occur in a horizontal plane, physical aids such as rulers and curve guides can be used very easily to assist in making pen drawings. We have noticed that, for some operations such as typing, looking at the original window on the vertical screen is often preferred. This is one of the motivations for maintaining both views. 
Second, a tangible interaction tool such as a brick element or enhanced physical paper can be used in the non-dominant hand to control the position and orientation of the VIEW, while interacting with the pen in the dominant hand. A more natural interaction, that closely resembles writing or sketching with a real pen on paper, can be accomplished in this way.

Third, a virtual paper or a sheet of real paper can be positioned on top of the VIEW. The user can write and draw on such a (real or virtual) sheet, using the image from the windows application as background. This may for instance assist in overdrawing parts of an existing document. A virtual paper may also capture an outlined portion of the VIEW, which may for instance be useful for creating collages of input material, in a way that resembles cutting and gluing in the real world. The VIEW may also be made transparent and overlaid on an existing virtual (or real) paper, so that this latter paper can be used as the background for the windows application. Aspects of this background image may be overdrawn or 'captured' by the windows application. Several different VIEWs can be combined in a similar way.

\subsection{Potential Electronic Paper improvements}

The Electronic Paper does not currently support 3D interaction. It may, however, be useful in future to enrich the system with the ability to manipulate and create 3D models. One possible way to support 3D interaction is an approach described by Subramanian (Subramanian, 2004). In this case the communication space of the Visual Interaction Platform (Fig. 1) is used to provide a perspective view of the horizontal workspace with the surface rendered 3D model(s), while the workspace can provide a top view of the scene or floor plan. This approach to 3D interaction does not allow 3D data creation. For a designer, the ability to create models is usually more important than the ability to explore existing data. So in order to introduce 3D in the design tool, we also should consider not only ways to manipulate the data but also ways to create new data. As a first step towards more intuitive 3D interaction, we have included optical tracking, using stereovision that was originally developed for the Personal Space Station (Mulder and van Liere, 2002). Some promising approaches for creating 3D shapes that do not require a 3D input device were proposed by Igarashi et al. (1999) in the Teddy tool and by Zeleznik et al. (1996) in the SKETCH prototype.

Architects often sketch in different environments such as the office, at home, outside (for example on a site), in the train, etc. (Gross and Yi-Luen Do, 1996). The Electronic Paper tool can be run on a portable Tablet PC, however, it is not yet integrated with a stationary version of the Electronic Paper. We are currently investigating flexible and natural ways of integrating the portable version of the tool with a stationary version of it.

\section{Conclusions}

In this paper we have presented the Electronic Paper prototype as the user interface to computer tools for early architectural design. The evolution of the presented tool has been mainly through a synthesis-by-analysis and iterative approach. User requirements were analyzed and initial system requirements for the tool were derived from these user 
requirements, while additional user requirements were derived from tool evaluation in different stages.

The prototype system can help in managing, storing and annotating images; managing, creating and editing sketches and can assist in re-drawing and over-drawing. It also preserves the naturalness of the traditional way of sketching. The Electronic Paper can be used on a mobile PC as well as in a stationary setting, so that architects can use the same tool in different situations. With the introduction of the VIEWs, the Electronic Paper prototype also naturally integrates pen\&paper interaction with standard windows applications. Although this combination may not be beneficial to all kinds of applications, it definitely has added value for some of them and improves the integration of different design stages. Some interesting directions in which we can extend the computer functionality of the Electronic Paper tool have also been identified.

The formal evaluation showed that the system does indeed support sketching in a way that is comparable with a traditional 'pen and paper' environment. Positioning of virtual components within the system needs to be improved if we want to more closely approximate the performance obtainable with positioning real objects. The evaluation of the Electronic Paper in a high-level architectural task has confirmed that the tool is usable and allows architects to not restrict their creativity and at the same time to have the power of the computer.

\section{Acknowledgements}

We thank Bernard Champoux and Andres Lucero for their graphical design work on different Electronic Paper prototypes. We thank the reviewers for insightful comments and suggestions.

\section{References}

Aliakseyeu, D., 2003. A Computer Support Tool for the Early Stages of Architectural Design. PhD Thesis, Eindhoven, The Netherlands, ISBN 90-386-1598-1.

Aliakseyeu, D., Martens, J.-B., 2001. Physical paper as user interface. In: Proceedings of Interact 2001, July 2001, Tokyo. IOS Press, pp. 680-681.

Aliakseyeu, D., Martens, J.-B., Subramanian, S., Vroubel, M., Wesselink, W., 2001. Visual interaction platform. In: Proceedings of Interact 2001, July 2001, Tokyo. IOS Press, pp. 232-239.

Bier, E.A., Stone, M.C., Pier, K., Buxton, W., DeRose, T.D., 1993. Toolglass and magic lenses: the see-through interface. In: Proceedings of the 20th Annual Conference on Computer Graphics and Interactive Techniques, New York, ACM Press, pp. 73-80.

Carroll, J.M., 1995. Scenario-based Design: Envisioning Work and Technology in System Development. Wiley, New York.

Dietz, P., Leigh, D., 2001. DiamondTouch: a multi-user touch technology. In: Proceedings of UIST'01, Orlando, FL. ACM Press, pp. 219-226.

Elliot, A., Hearst, M., 2000. How large should a digital desk be? qualitative results of a comparative study. In: Extended abstracts of CHI'2000, April 2000, The Hague, ACM Press, pp. 165-166.

Elliot, A., Hearst, M., 2002. A comparison of the affordances of a digital desk and tablet for architectural image tasks. Journal Human-Computer Studies, 56, 173-197. 
Elrod, S., Pier, K., Tang, J., Welch, B., Bruce, R., Gold, R., Goldberg, D., Halasz, F., Janssen, W., Lee, D., McCall, K., Pedersen, E., 1992. Liveboard: a large interactive display supporting group meetings, presentations, and remote collaboration. In: Proceedings of CHI'92, Monterey, CA. ACM Press, pp. 599-607.

Fitzmaurice, G.W., Buxton, W., 1997. An empirical evaluation of graspable user interfaces: towards specialized, space-multiplexed input. In: Proceedings of CHI'97, Atlanta, GA. ACM Press, pp. 43-50.

Fitzmaurice, G.W., Ishii, H., Buxton, W., 1995. Bricks: laying the foundations for graspable user interfaces. In: Proceedings of CHI'95, Denver, May 1995. ACM Press, pp. 442-449.

Fitzmaurice, G., Balakrishnan, R., Kurtenbach, G., Buxton, B., 1999. An exploration into supporting artwork orientation in the user interface. In: Proceedings of CHI'99, May 1999, Los Angeles. ACM Press, pp. 167-174.

Fox, A., Johanson, B., Hanrahan, P., Winograd, T., 2000. Integrating information appliances into an interactive workspace. IEEE Computer Graphics and Applications, May/June 2000, IEEE, pp.54-65.

Gribnau, M.W., 1999. Two-handed Interaction in Computer-supported 3D Conceptual Modelling. Doctoral dissertation, Delft University of Technology, The Netherlands.

Gross, M., Yi-Luen Do, E., 1996. Ambiguous intentions: a paper-like interface for creative design. In: Proceedings of UIST'96, November 1996, Seattle. ACM Press, pp. 183-192.

Guiard, Y., 1987. Asymmetric division of labor in human skilled bimanual action: the kinematic chain as a model. Journal of Motor Behavior, 19 (4), 486-517.

Hinckley, K., 1997. Haptic Issues for Virtual Manipulation. PhD Thesis, Department of Computer Science, University of Virginia.

Holman, D., Vertegaal, R., Altosaar, M.,Troje, N., Johns, D., 2005. PaperWindows: interaction techniques for digital paper. In: Proceedings of CHI'05, Portland, Oregon. ACM Press, pp. 591-599.

Igarashi, T., Matsuoka, S., Tanaka, H., 1999. Teddy: a sketching interface for 3D Freeform design. In: Proceedings of SIGGRAPH'99, July 1999, Los Angeles. ACM Press, pp. 409-416.

Ishii, H., Underkoffler, J., Chak, D., Piper, B., Ben-Joseph, E., Yeung, L., Kanji, Z., 2002. Augmented urban planning workbench: overlaying drawings, physical models and digital simulation. In: Proceedings of ISMAR 2002. IEEE.

Klemmer S.R., Newman, M.W., Farrell, R., Bilezikjian, M., Landay, J.A., 2001. The designers' outpost: a tangible interface for collaborative web site. In: Proceedings of UIST'01, Orlando, FL. ACM Press, pp. 1-10.

Landay, J.A., Myers, B.A., 2001. Sketching interfaces: toward more human interface design. Computer, March 2001. IEEE, pp. 56-64.

Lawson, B., 1999. 'Fake' and 'real' creativity using computer aided design: some lessons from Herman Hertzberger. In: Proceedings of Creativity \& Cognition'99, October 1999, Loughborough, ACM Press, pp. 174-180.

Mackay, W., Fayard A.L., 1998. Designing interactive paper: lessons from three augmented reality projects. In: Proceedings of IWAR'98, Natick, MA. A.K Peters, pp. 81-90.

Martens, J.-B., Aliakseyeu, D., de Pijper, J.-R., 2004. VIEWs: visual interaction enriched windows. In: Proceedings of EUSAI'04, Eindhoven, The Netherlands. Springer, pp. 255-266.

McGee D.R., Cohen P.R., Wesson R.M., Horman, S., 2002. Comparing paper and tangible, multimodal tools. In: Proceedings of CHI'02, Minneapolis, MN. ACM Press, pp. 407-414.

Moran, T.P., Saund, E., Melle, W. van, Bryll, R., Gujar, A.U., Fishkin, K.P., Harrison, B.L., 1999a. The ins and outs of collaborative walls: demonstrating the collaborage concept. In: Proceedings of CHI'99, Pittsburgh, PA. ACM Press, pp. 192-193.

Moran, T.P., Saund, E., Melle, W. van, Bryll, R., Gujar, A.U., Fishkin, K.P., Harrison, B.L., 1999b. Design and technology for collaborage: collaborative collages of information on physical walls. In: Proceedings of UIST'99, Asheville, NC. ACM Press, pp. 197-206.

Mulder, J.D., van Liere, R., 2002. The personal space station: bringing interaction within reach. In: Richer, S., Taravel, B., (Eds.), Proceedings of the Virtual Reality International Conference, VRIC 2002, p. 73-81.

Mynatt, E.D., Igarashi, T., Edwards, W.K., LaMarca, A., 1999. Flatland: new dimensions in office whiteboards. In: Proceedings of CHI'99, Pittsburgh, PA. ACM Press, pp. 346-353.

Novitski, B., 2002. AutoDesk goes conceptual. Architecture Week, Page T1.1. 08. Retrieved May 14, 2001, from http://www.architectureweek.com/2002/0508/tools_1-1.html.

Pedersen, E.R., McCall, K., Moran, T.P., Halasz, F.G., 1993. Tivoli: an electronic whiteboard for informal workgroup meetings. In: Proceedings of CHI'93, Amsterdam, The Netherlands. ACM Press, pp. 391-398. 
Piccolotto, A., 1998. SketchPad +. Architectural Modeling through Perspective Sketching on Pen-based Display. Master of Science Thesis, Program of Computer Graphics, Cornell University.

Piper, B., Ratti, C., Ishii, H., 2002. Illuminating clay: a 3-D tangible interface for landscape analysis. In: Proceedings of CHI'02, Minneapolis, MN. ACM Press, pp. 355-362.

Rauterberg, M., Bichsel, M., Meier, M., Fjeld, M., 1997. A gesture based interaction technique for a planning tool for construction and design. In: Proceeding of IEEE International Workshop on Robot and Human Communication 97. IEEE, pp. 212-217.

Rekimoto, J., 1997. Pick-and-Drop a direct manipulation technique for multiple computer environments. In: Proceedings of UIST 1997, Banff, Alberta, Canada. ACM Press, pp. 31-39.

Rekimoto, J., 2002. SmartSkin: an infrastructure for freehand manipulation on interactive surfaces. In: Proceedings of CHI'02, Minneapolis, MN. ACM Press, pp. 113-120.

Robinson, J., Robertson, C., 2001. The LivePaper system: augmenting paper on an enhanced tabletop. Computers \& Graphics, 25, 731-743.

Segers, N.M., 2002. Towards a data-structure that can handle ambiguous information in a computer-aided tool for the early phase of architectural design. In: Timmermans, H.J.P., Vries, B. de (Eds.), Design \& Decision Support Systems in Architecture-Proceedings of the Sixth International Conference, July 7-10, 2002. Ellecom, pp. 339-351.

Segers, N.M., 2004. Computational Representations of Words and Associations in Architectural Design. PhD thesis, Eindhoven University of Technology, Eindhoven, The Netherlands, ISBN: 90-6814-576-2.

Subramanian, S., 2004. Tangible Interfaces for Volume Navigation. PhD Thesis, Eindhoven University of Technology, Eindhoven, The Netherlands.

Ullmer, B., Ishii, H., 1997. The metaDESK: models and prototypes for tangible user interfaces. In: Proceedings of UIST'97, Banff, Alberta, Canada, pp. 223-232.

Ullmer, B., Ishii, H., 2001. Emerging frameworks for tangible user interfaces. In: Varrol, John M. (Ed.), HumanComputer Interaction in the New Millennium. Addison-Wesley, Reading, MA, pp. 579-601.

Wellner, P., 1991. The DigitalDesk calculator: tangible manipulation on a desk top display. In: Proceedings of UIST'91, November 19991, Hilton Head. ACM Press, pp. 27-33.

Zeleznik, R., Herndon, K., Hughes, J. (1996) SKETCH: an interface for sketching 3D scenes. In: Proceedings of SIGGRAPH'96, August 1996. ACM Press, pp. 163-170. 